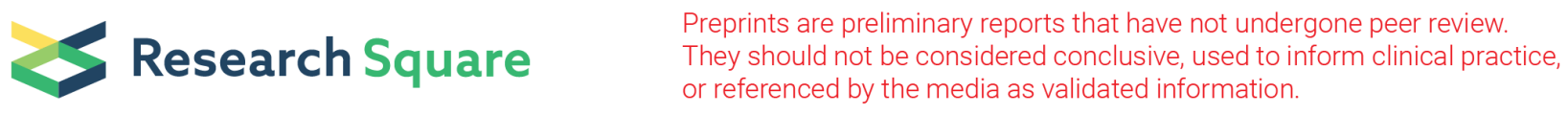

\title{
Degradation Dynamics of Quantum Dots in White LED Applications
}

Hsiao-Chien Chen

Chang Gung University

Cher Ming Tan ( $\nabla$ cmtan@cgu.edu.tw)

Chang Gung University

Abdul Shabir

Chang Gung University

Preetpal Singh

Chang Gung University

Kai Wang

Southern University of Science and Technology

\section{Research Article}

Keywords: Degradation Dynamics, Quantum Dots, White LED Applications, blue LED chip, light source, degradation, oxidation

Posted Date: August 17th, 2021

DOI: https://doi.org/10.21203/rs.3.rs-795820/v1

License: (c) (i) This work is licensed under a Creative Commons Attribution 4.0 International License. Read Full License

Version of Record: A version of this preprint was published at Scientific Reports on December 1st, 2021. See the published version at https://doi.org/10.1038/s41598-021-02714-0. 


\section{Abstract}

Quantum dots (QDs) are being investigated in a hybrid white light LED structure which inculcates phosphor in the package with blue LED chip as light source. Upon prolong operation, degradation of CdS QDs is observed which can limit its practical applications. The degradation includes intensity reduction as well as emitted wavelength shift of the white light. Three stages of degradation are observed, namely an enhancement state where light intensity is observed to increase, followed by a rapid degradation stage where light intensity decreases rapidly, and finally a slower degradation state where light intensity degradation rate slows down and continue till end of test.

In this work, the degradation of CdS QDs is analyzed using various material analysis tools. Density functional theory (DFT) calculation is performed to confirm the spectroscopy results. It is found that the time evolving degradation of QDs begins from the oxidation of sulfur vacancy of CdS QDs by nearby oxygen atoms due to the imperfection of the protective coating around the QDs in combination of the presence of blue light, and this oxidation renders a transformation of $\mathrm{CdS}$ into $\mathrm{CdO}$ at the initial state. The final stage is the formation of $\mathrm{CdSO}_{4}$ via some intermediate processes.

\section{Introduction}

Owing to their size-dependent optical properties ${ }^{[1,2]}$, semiconductor quantum dots (QDs) have been receiving increasing research interest recently. Their emission wavelength varies as a function of particle size ${ }^{[3,4]}$. This ability to tune the optical properties through variation in size and its narrow emission spectrum, QDs are widely employed for light conversion process in LEDs in recent decades ${ }^{[5,6]}$.

The combination of long lifetime and color stability of phosphor and narrow emission peak of QDs, along with mature blue LEDs can be a future for the night vision cameras and aviation technology ${ }^{[5,6]}$. Generally, a blue LEDs is used as light source followed by a green phosphor and red QDs layer on the top to produce white light. Such LED is named as QDLED in this work.

Both the materials (QDs and phosphor) have their own strengths and weaknesses as studied separately in the past ${ }^{[7-10]}$. QDs and phosphor's performances as light down convertors have been individually studied by many workers ${ }^{[11-13]}$. However, the performance and durability of their integration into LED package is yet to be studied. Before embarking on the prospective applications of QDLED, it is important to evaluate the performance durability of QDLEDs in lighting applications under various operating conditions, especially when the QDs are subjected under blue light illumination along with ambient moisture and temperature, which corresponds to the condition in outdoor environment applications.

A packaged LED structure is fabricated with blue LED as a light source and QDs- phosphor layers as the down conversion materials. This work analyzes the QDs degradation behavior systematically and its impact on the overall light output when it is used in a hybrid structure in collaboration with phosphor to produce white light. Various unique light output degradation trends are observed, and investigations of their causes are performed for better understanding of these behaviors. QD emission parameters such as peak wavelength and intensity are studied as peak wavelength changes can result from a change in the size of the nanoparticles ${ }^{[14,15]}$. In this work, more attention is given towards the QD characteristics and its impact on the overall LED light output as phosphor is already a well-studied material in packaged LEDs.

\section{Results And Discussion}

The degree of lumen degradation over test time is shown in Fig. 2, and the overall luminescence intensity weaken with test time, revealing degradation occurs in the duration of working condition. According to the lumen variation shown in Fig. 2, the degradation can be divided into three stages, namely enhancement stage, rapid degradation stage and slower degradation stage, respectively. Stage 1 is termed as enhancement state where light intensity is observed to increase for the first 18 hours of test. Stage 2 is termed as rapid degradation stage where light intensity decreases rapidly from 18 to 220 hours of test. Stage 3 is termed as slower degradation state or stable state where light intensity degradation rate slows down. This stage continues till the end of the test of 900 hours and the light intensity degrades to around $80 \%$ of its initial value. Each stage has different characteristics and hence they are likely to have different causes and implications on the overall reliability and efficiency of the structure.

Lumen degradation can be due to the degradation of either blue LED, phosphor, and QDs or the combination of either of these. To clarify the sources of the degradation behaviors, light intensity variations with respect to its wavelengths are separated as shown Fig. 3 . It can be observed that the blue LED peak intensity around $450 \mathrm{~nm}$ varies with test time. However, it is known that blue LED chip fabrication is a mature technology and does not degrade easily especially under normal ambient conditions as performed in this test ${ }^{[16]}$. To verify the health of the blue LED chips, I-V curves of the blue LED chips is measured with a typical curve shown in Fig. 4. No shift in the I-V curve is observed when tested up to 268 hours, implying that the LED chips are intact and do not undergo any degradation, as expected. Therefore, the blue peak intensity variation can be attributed to two factors. First, the loss of blue light may be due to the light scattering caused by moisture that has penetrated inside the encapsulant in the early hours of the test in the similar way as reported in literature ${ }^{[17]}$. However, in view of the $44 \% \mathrm{RH}$ in the operating condition at room temperature, such diffusion of moisture into silicone encapsulation is unlikely in this short initial period. Therefore, the blue light intensity variation is likely to be due to the changes in the structure of phosphors or CdS QDs which can increase the transmittance or absorption of blue light.

According to the conversion efficiency plots which are computed as the ratio of blue light intensity and light intensity from phosphor or QDs as shown in Fig. 5, and the blue light is used as a reference since the blue LED chip is intact, the conversion efficiency of QDs and phosphor vary differently, and consequently the variation in the output light intensity vary differently in different stages. It can be observed that the light intensity of phosphor decreases slightly during the first 200 hours and then reaches a steady state. The $13 \%$ decreases in the phosphor conversion efficiency observed might be attributed to

Page 2/12 
the slight increase of temperature and electro-thermal stress ${ }^{[18,19]}$, and Fig. 6 indeed shows a rise in temperature for LED before and after the test, with the temperature increased from $35.6^{\circ} \mathrm{C}$ to $37.4^{\circ} \mathrm{C}$ and remain constant subsequently. Let us now focus on the degradation of QDs.

In the enhancement stage, light conversion efficiency of QDs increases during the initial 6 hours and followed by a rapid degradation in the subsequently. The increase in the light intensity from QDs might be attributed to the oxygen adsorption on the surface of QDs which will suppress the inherent surface defect and increase the QDs luminescence as reported by Singh et.al ${ }^{[20]}$. On the other hand, presence of oxygen atoms can also render photo-oxidation of QD, decreasing the QDs luminescence ${ }^{[21]}$. Therefore, a competition between passivation of QDs surface defects and photo-oxidation exist, and this could lead to an increase in QDs light output intensity at the first 6th hours followed by the subsequent decrease till 18th hours.

Energy-dispersive X-ray spectroscopy (EDS) mapping taken using JEOL-JSM SEM/OXFORD EDS with SEI mode is used to examine the composition ratio of elements of the QD, and the results are shown in Fig. 7. Although the shell structure of ZnS is implemented as the protective layer, it can be seen that the distribution of ZnS in not uniform, indicating incomplete surface protection that allow the absorption of oxygen and the oxidation of QD in the presence of light. This photo-oxidation is also confirmed by Raman spectroscopy by UniNano UNIDRON-A Raman microscopy system. Two characteristic peaks of fresh CdS QDs are observed at 299 and $597 \mathrm{~cm}^{-1}$, which are assigned to the fundamental optical phonon mode (LO) and the first overtone mode (2LO) of CdS ${ }^{[22,23]}$ (Fig. 8). The characteristic bands of CdO are also found at $529 \mathrm{~cm}^{-1}$, broad band at 350 to $500 \mathrm{~cm}^{-1}$ and broad band at 750 to $950 \mathrm{~cm}^{-1}$ as shown in Fig. $8^{[24,25]}$. It is widely known that this defect structure would trap the photo-excited electron and further hinder radiative recombination, thus decreasing the $\mathrm{PL}$ intensity of QDs ${ }^{[26,27]}$. The formation of CdO at S-vacancy reduces the ability of electron trap, resulting in an increase in the PL emission of CdS QDs, which is consistent with the result of the enhancement stage. The intensity of CdO peak further increases at 18 hours, but the increase in intensity is not obvious at 220 hours, implying that major oxidation of CdS to CdO is within the first 18 hours. In addition, a new weak band at $1088 \mathrm{~cm}^{-1}$ is detected at 18 hours, which is assigned to the vibration of the $\mathrm{SO}_{4}{ }^{2-}$ group of $\mathrm{CdSO}_{4}$, and the strength of the band increases with time, indicating the amount of $\mathrm{CdSO}_{4}$ gradually increases during the process ${ }^{[28]}$.

Comparing the weight ratio of $\mathrm{Cd}$ to S element, the values of $\mathrm{CdS}$ at 0,18 and 220 hours are 2.31, 2.40 and 2.23, where is an insignificant change. However, there is an increase in the oxygen element content with the test time, which is attributed to the formation of $\mathrm{CdO}$ and $\mathrm{CdSO}_{4}$ as demonstrated by Raman spectroscopy. To understand the implication of this information, DFT based simulation is performed.

It is reported that the oxidation of QDs is mainly dominated by oxygen rather than $\mathrm{H}_{2} \mathrm{O}^{[29]}$, only oxygen is involved in our DFT based simulation. The structure for tracing the MEP is designed by assuming that the CdS structure already has a sulphur vacancy resulting in $16 \mathrm{Cd}$ atoms and $15 \mathrm{~S}$ atoms in the periodic cell. This sulphur vacancy creates three dangling bonds in the neighboring Cd atoms allowing adsorption of oxygen atoms to form CdO. With this assumption, we introduce two $\mathrm{O}_{2}$ molecules near the defect site of the CdS structure. The geometry optimization of the DFT simulation indicates direct adsorption of $\mathrm{O}_{2}$ molecules in the defect sites thereby forming $\mathrm{Cd}-\mathrm{O} / \mathrm{Cd}-\mathrm{O}_{2}$ bonds as shown in Fig. 9.

The first intermediate of the degradation process shows the formation of $\mathrm{CdO} \mathrm{O}^{*}$ and $\mathrm{SO}_{2}$ molecules in an endergonic process as the $\Delta \mathrm{G}$ value is positive. This indicates that the formation of this intermediate is non-spontaneous. However, in the presence of an external energy source such as blue light, it is a reachable step because the required energy is $250 \mathrm{~kJ} \mathrm{~mol}^{-1}$ and the energy supplied by the blue light is $254 \mathrm{~kJ} \mathrm{~mol}^{-1}$. However, once the first intermediate is attained, it is quickly dissociated into other intermediates. The transition state of the dissociation reaction is exergonic and more spontaneous than the previous intermediate as the $\Delta \mathrm{G}$ value is negative.

The transition state indicates dissociation of an O-atom from the $\mathrm{SO}_{2}$ molecule, thus creating a $\mathrm{SO}^{*}$ complex. The formation of $\mathrm{SO}_{2}$ and $\mathrm{SO}^{*}$ complex induce further S-vacancy in the CdS structure which was seen to be passivated by the $\mathrm{O}^{*}$ ion forming $\mathrm{CdO}$ as shown in Fig. 9b-c. Due to the additional defects induced by $\mathrm{SO}_{2}$ formation, more $\mathrm{Cd}$ dangling bonds are created in the structure. These vacant sites are passivated by oxygen with the co-ordination number of 0 -atom in the CdO complex varied between 3 and 4 as shown in Fig. 9c. However, the co-ordination number of Cd atoms near the defect site changed from 4 to 5 which characterizes the transition state structure as the co-ordination number for $\mathrm{Cd}(\mathrm{II})$ atoms are 4 while for $\mathrm{Cd}(\mathrm{IV})$ atoms is 6 . The bond length of the $\mathrm{Cd}-\mathrm{O}$ bond length was consistent at $2.27 \AA$, while the $\mathrm{Cd}-\mathrm{Cd}$ bond formed in the transition state structure has a bond length of $3.24 \AA$.

Even though this is a spontaneous intermediate, the atomic arrangement is unstable and this leads to the final stage of the degradation via bond rearrangement. The formation of a $\mathrm{CdSO}_{4}$ complex in the $\mathrm{CdS}$ nanostructure by oxidation is also an exergonic reaction with a highly negative $\Delta \mathrm{G}$. The spontaneity of this reaction complex indicates formation of $\mathrm{CdSO}_{4}$ due to sulphur vacancies in presence of $\mathrm{O}_{2}$ is a favorable reaction.

Our simulation confirms the constant sulfur concentration as the intermediate of $\mathrm{SO}_{2}$ would quickly transform into $\mathrm{CdO}^{*}, \mathrm{SO}^{*}$ and $\mathrm{CdSO}_{4}$ that prevents the loss of sulphur element, which agrees with the constant weight ratio of Cd element to S element from our EDS mapping. On the other hand, the incorporation of oxygen element in the $\mathrm{CdS}$ structure to form $\mathrm{CdO}$ and $\mathrm{CdSO}_{4}$, causes an increase in the oxygen element as observed in our EDS map.

Figure 10 shows the variations of absorption and PL spectra of CdS QDs with time. In the case of QDs at 18 and 220 hours of working conditions, the absorption behavior between $500 \mathrm{~nm}$ to $580 \mathrm{~nm}$ are different from the fresh QDs. The quantum confinement produces complex structure of hole quantized states due to the mixing valences sub-bands, results in the formation of various interband optical transitions involved in $1 \mathrm{~S}$ or $1 \mathrm{P}$ electron state, thus exhibiting a broad absorption band in the range of $500 \mathrm{~nm}$ to $580 \mathrm{~nm}^{[30]}$. It means that the difference of absorption behavior within $500 \mathrm{~nm}$ to $580 \mathrm{~nm}$ is attributed to the change of the electronic structure of $\mathrm{CdS}$ QDs involved in interband optical transitions due to formation of $\mathrm{CdO}^{-\mathrm{CdS}}$, $\mathrm{CdSO}{ }_{4}$-CdS and intermediate species which are predicted in our simulation and demonstrated by Raman spectroscopy. The absorption spectrum of fresh CdS QDs shows a major peak at $602 \mathrm{~nm}$ which is the dominating excitonic transitions from ground electronic sub-band to the ground hole sub-band ${ }^{[31,32]}$. It shows a blue shift to $600 \mathrm{~nm}$ and $597 \mathrm{~nm}$ at 18 and 220 hours, resulting in the blue shift of emission peak from $628 \mathrm{~nm}$ to $627 \mathrm{~nm}$ and $625 \mathrm{~nm}$, respectively. The results of the

Page $3 / 12$ 
blue shift of PL emission reveals the decrease of particle size due to the photo-oxidation, which is well in agreement with the quantum size effect and the result of the packaged LED ${ }^{[33]}$.

It is also observed that a rapid luminescence degradation of QDs is accompanied by a continuous blue shift in QDs peak wavelength from 18 to 364 hours of test (Fig. 11). This stage can be considered as an extension of the end of the first stage where photo-oxidation mechanism becomes dominating over surface defect passivation process for QDs because the blue to red intensity ratio in this stage is higher than that in enhancement stage and that the intensity of the

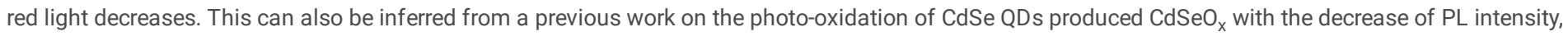
reduction of particle size and blue shift of $\mathrm{PL}$ emission ${ }^{[21]}$, and the formation of $\mathrm{CdSO}_{4}$ is also the final product of our simulation. In other words, the reduction of QDs luminescence and blue shift of QDs emission in rapid degradation stage is attributed to the effect of photo-oxidation.

In the final stage, a negligible overall LED light output change is observed with corresponding slight changes in the QDs luminescence. It seems that the surface oxidation of QDs that resulted in a rapid decrease in wavelength during second stage is slowing down as it enters into this stage. This phenomenon is generally observed in the oxidation of QDs, where the outmost layer of QDs is used for sacrificial oxidation to form a protective oxide layer ${ }^{[34]}$. This oxide layer could effectively prevent further oxidation of interior QDs. As shown in Fig. 5, the variation of blue to red intensity ratio tends to stabilize, suggesting the surface oxidation of the QDs is nearly complete. The existence of the shell layer can effectively slow down the deep oxidation of QDs ${ }^{[35]}$.

\section{Conclusions}

The white light packaged LED is fabricated by the hybrid structure of blue LED, phosphor and QDs in this study. The result of simulation and spectroscopies clearly indicate the impact of oxygen atoms on the durability of CdS QDs in the presence of blue light which transforms the CdS QDs into CdO-CdS and $\mathrm{CdSO}_{4}$-CdS QDs. This transformation causes lumen degradation and color shift. Our results show that the defect structure is the starting position of QDs oxidation. While oxygen atoms are hard to eliminate, the intensity of blue light and the defects of the protective coating over QDs are vital to the durability of QDs in white light LED applications.

\section{Methods}

\section{Experimentation}

5 LED samples are prepared using blue LED chips as their illumination sources. The schematic of the LEDs structure and its corresponding luminescence plot is shown in Fig. 1, which is constructed by blue LED, phosphor for emitting green light and CdS QDs for emitting red light. The three parts of structure contribute a wide emitting spectroscopy and result in a white light emission (Fig. 1).

To investigate the stability of these LED devices, experimental duration of the LEDs operating under ambient temperature of $295.8 \mathrm{~K}$ and relative humidity of approximately $44 \% \mathrm{RH}$ is set to 900 hours unless a $20 \%$ of lumen decay is detected earlier.

An initial set of measurements for all the samples are done to serve as reference baseline for each test sample. These measurements include electrical and lumen measurements. Lumen measurement is done using Mentor's TeraLED integrating sphere and Electrical measurement is done using Keithley 2461 Source meter. The blue LED in each sample is powered with 30-mA constant current by Keithley power supply model $2651 \mathrm{~A}$. The optical parameters, namely light intensity and peak wavelength are monitored every 3 hours.

\section{Atomic Scale Modelling and Simulation Methodology}

In order to understanding the detail degradation mechanisms as observed experimentally as will be reported later, atomic scale modeling is employed. It has been established that CdS nanostructures have a dominant hexagonal crystal structure ${ }^{[36,37]}$. With this reference, we used a P63mc space group CdS structure from Medea's Infomatica database ${ }^{[38]}$ and extended it into a $2 \times 2 \times 2$ supercell consisting of $16 \mathrm{Cd}$ and $\mathrm{S}$ atoms respectively.

The density functional theory (DFT) calculations are carried out by projector-augmented wave (PAW) method ${ }^{[39]}$ as implemented in the Vienna Ab-inito Simulation Package provided by Medea (Medea-VASP) ${ }^{[38,40]}$. The interactions are described using the Generalized Gradient Approximation with Perdew-BurkeErnzerhof (GGA-PBE) exchange-correlation functional ${ }^{[41]}$. The atomic orbitals are defined by plane-wave basis sets with cut-off energies of 300 eV[42]. Reciprocal space projection operators are used and the convergence criterion for self-consistent field calculations is set to $10^{-5} \mathrm{eV}$ between consecutive steps. The convergence criterion for atomic forces of the systems is set to be less than $0.002 \mathrm{eV} \mathrm{A}^{-1}$. The Brillouin zone is sampled at $2 \times 2 \times 1 \mathrm{gamma}$ centered $\mathrm{k}$ points where the actual k-point spacing is $0.439 \times 0.439 \times 0.465$ per Angstrom.

The transition state search (TSS) module of the MedeA software is used to observe the energy changes between the initial and final structures ${ }^{[38,43]}$. In the TSS module, we employ Nudged elastic band (NEB) method ${ }^{[43,44]}$ to map the minimum energy path (MEP) between the initial and the final systems by calculating the free energy $(\Delta G)$ barriers at different reaction stages. The stage closest to a saddle point is allowed to climb up into the saddle point (cNEB method) if the largest force on an atom is smaller than $1.0 \mathrm{eV} \AA^{-1}$. The Self Consistent Field (SCF) is calculated by RMM_DIIS minimizer method [42, 45]. Elements of the inverse Hessian are initially set to $0.001 \AA^{2} \mathrm{eV}^{-1}$ and linear interpolation is used to create the reaction intermediates. Reaction coordinate is the normalized coefficient of linear interpolation with translation criterion of 0.5 .

\section{Declarations}


The authors acknowledge support from the Ministry of Science and Technology, Taiwan (Contract Nos. MOST 108-2221-E-182-027- and 109-2222-E-182 -002 MY3).

\section{Author Contributions}

C.M.T and H.C.C conceived the idea of the project. K.W supported the materials and packaged LED. H.C.C, A.S and P.S designed and performed the experiments. A.S performed ab initio simulations. C.M.T, H.C.C and A.S analyzed the experimental data and discussed the results. H.C.C and A.S wrote the manuscript.

\section{Competing Financial Interests}

The authors declare no known competing financial interests.

\section{References}

[1] B. S. Mashford, M. Stevenson, Z. Popovic, C. Hamilton, Z. Zhou, C. Breen, J. Steckel, V. Bulovic, M. Bawendi, S. Coe-Sullivan, P. T. Kazlas, Nat. Photonics2013, 7, 407.

[2] S. Coe-Sullivan, W. K. Woo, J. S. Steckel, M. Bawendi, V. Bulović, Org. Electron.2003, 4, 123.

[3] Device and Method for Emitting Output Light Using Quantum Dots and Non-Quantum Fluorescent Material, 2006.

[4] Q. Sun, Y. A. Wang, L. S. Li, D. Wang, T. Zhu, J. Xu, C. Yang, Y. Li, Nat. Photonics2007, 1, 717.

[5] S. Bera, S. B. Singh, S. K. Ray, in J. Solid State Chem., Academic Press, 2012, pp. 75-79.

[6] Y. Yang, Y. Zheng, W. Cao, A. Titov, J. Hyvonen, J. R. Manders, J. Xue, P. H. Holloway, L. Qian, Nat. Photonics2015, 9, 259.

[7] Y. Li, A. Rizzo, R. Cingolani, G. Gigli, Adv. Mater.2006, 18, 2545.

[8] R. Xie, D. Battaglia, X. Peng, J. Am. Chem. Soc.2007, 129, 15432.

[9] S. Nishiura, S. Tanabe, K. Fujioka, Y. Fujimoto, in Opt. Mater. (Amst)., Elsevier B.V., 2011, pp. 688-691.

[10] C. M. Tan, P. Singh, W. Zhao, H. C. Kuo, Sci. Rep.2018, 8, 2452.

[11] W. S. Song, J. H. Kim, J. H. Lee, H. S. Lee, Y. R. Do, H. Yang, J. Mater. Chem.2012, 22, 21901.

[12] H. V. Demir, S. Nizamoglu, T. Erdem, E. Mutlugun, N. Gaponik, A. Eychmüller, Nano Today2011, 6, 632.

[13] Y. Narukawa, I. Niki, K. Izuno, M. Yamada, Y. Murazaki, T. Mukai, Japanese J. Appl. Physics, Part 2 Lett.2002, 41, L371.

[14] D. D. W. Grinolds, P. R. Brown, D. K. Harris, V. Bulovic, M. G. Bawendi, Nano Lett.2015, 15, 21.

[15] W. M. Girma, M. Z. Fahmi, A. Permadi, M. A. Abate, J. Y. Chang, J. Mater. Chem. B2017, 5, 6193.

[16] C. Wang, E. Yifeng, L. Fan, Z. Wang, H. Liu, Y. Li, S. Yang, Y. Li, Adv. Mater.2007, 19, 3677.

[17] E. D. Spoerke, B. A. Connor, D. V. Gough, B. B. McKenzie, G. D. Bachand, Part. Part. Syst. Charact.2014, 31, 863.

[18] MedeA Software | Materials Design Inc, https://www.materialsdesign.com/medea-software, accessed: May, 2021.

[19] P. E. Blöchl, C. J. Först, J. Schimpl, Bull. Mater. Sci.2003, 26, 33.

[20] M. Zhu, M. Yu, M. Xia, B. Li, P. Yu, S. Gao, Z. Qi, L. Liu, Y. Chen, H. Guan, in Proc. ACM Symp. Appl. Comput., 2011, pp. 554-559.

[21] G. K. H. Madsen, Phys. Rev. B - Condens. Matter Mater. Phys.2007, 75, 195108.

[22] G. Kresse, J. Furthmüller, Phys. Rev. B - Condens. Matter Mater. Phys.1996, 54, 11169.

[23] G. Henkelman, G. Jóhannesson, H. Jónsson, in Theor. Methods Condens. Phase Chem., Kluwer Academic Publishers, 2005, pp. 269-302.

[24] G. Henkelman, B. P. Uberuaga, H. Jónsson, J. Chem. Phys.2000, 113, 9901.

[25] T. Rohwedder, R. Schneider, T. Rohwedder, · R Schneider, S. Ma, R. Schneider, J Math Chem2011, 49, 1889.

[26] N. Jiang, J. Zou, C. Zheng, M. Shi, W. Li, Y. Liu, B. Guo, J. Liu, H. Liu, X. Yin, Appl. Sci.2018, 8, 1940.

[27] C. M. Tan, B. K. Eric Chen, G. Xu, Y. Liu, Microelectron. Reliab.2009, 49, 1226. 
[28] E. Jung, J. H. Ryu, C. H. Hong, H. Kim, J. Electrochem. Soc.2011, 158, H132.

[29] G.-H. Ryu, H.-Y. Ryu, J. Opt. Soc. Korea, Vol. 19, Issue 3, pp. 311-3162015, 19, 311.

[30] P. Singh, C. M. Tan, W. Zhao, H. C. Kuo, IEEE Trans. Device Mater. Reliab.2019, 19, 290.

[31] W. G. J. H. M. Van Sark, P. L. T. M. Frederix, D. J. Van den Heuvel, H. C. Gerritsen, A. A. Bol, J. N. J. Van Lingen, C. De Mello Donegá, A. Meijerink, J. Phys. Chem. B2001, 105, 8281.

[32] F. Ballipinar, A. C. Rastogi, J. Photonics Energy2018, 8, 1.

[33] A. G. Milekhin, L. L. Sveshnikova, T. A. Duda, N. V. Surovtsev, S. V. Adichtchev, D. R. T. Zahn, JETP Lett.2008, 88, 799.

[34] H. Güney, Vacuum2019, 159, 261.

[35] S. G. Choi, L. M. Gedvilas, S. Y. Hwang, T. J. Kim, Y. D. Kim, J. Zúñiga-Pérez, V. Muñoz Sanjosé, J. Appl. Phys.2013, 113, 183515.

[36] M. Micheel, B. Liu, M. Wächtler, Catalysts2020, 10, 1.

[37] Z. Chen, P. Z. Zhang, Y. Zhou, X. Zhang, X. Liu, Z. Hou, J. Tang, W. Li, J. Phys. Chem. Lett.2020, 11, 10354.

[38] Infrared and Raman Characteristic Group Frequencies: Tables and Charts - George Socrates - Google Books, https://books.google.com.tw/books? $\mathrm{hl}=$ en\&lr=\&id=LDoAAjMnwEIC\&oi=fnd\&pg=PP21\&dq=George+S+2004+Infrared+and+Raman+Characteristic+Group+Frequencies:+Tables+and+Charts,+3rd+Ec S 2004 Infrared and Raman Characteristic Group Frequencies\%3A Tables and Charts\%2C 3rd Edition\&f=false, accessed: May, 2021.

[39] C. W. Yeh, G. H. Chen, S. J. Ho, H. S. Chen, ACS Appl. Nano Mater.2019, 2, 5290.

[40] V. I. Klimov, Annu. Rev. Phys. Chem.2007, 58, 635.

[41] D. U. Lee, D. H. Kim, D. H. Choi, S. W. Kim, H. S. Lee, K.-H. Yoo, T. W. Kim, Opt. Express2016, 24, A350.

[42] Y. Wang, V. D. Ta, Y. Gao, T. C. He, R. Chen, E. Mutlugun, H. V. Demir, H. D. Sun, Adv. Mater.2014, 26, 2954.

[43] W. William Yu, X. Peng, Formation of High-Quality CdS and Other II \pm VI Semiconductor Nanocrystals in Noncoordinating Solvents: Tunable Reactivity of Monomers ${ }^{\star *}, 2002$.

[44] L. Huang, Z. Li, C. Zhang, L. Kong, B. Wang, S. Huang, V. Sharma, H. Ma, Q. Yuan, Y. Liu, G. Shen, K. Wu, L. Li, Chem. Sci.2019, 10, 6683.

[45] V. John, in Introd. to Eng. Mater., Palgrave Macmillan UK, 1992, pp. 419-433.

\section{Figures}
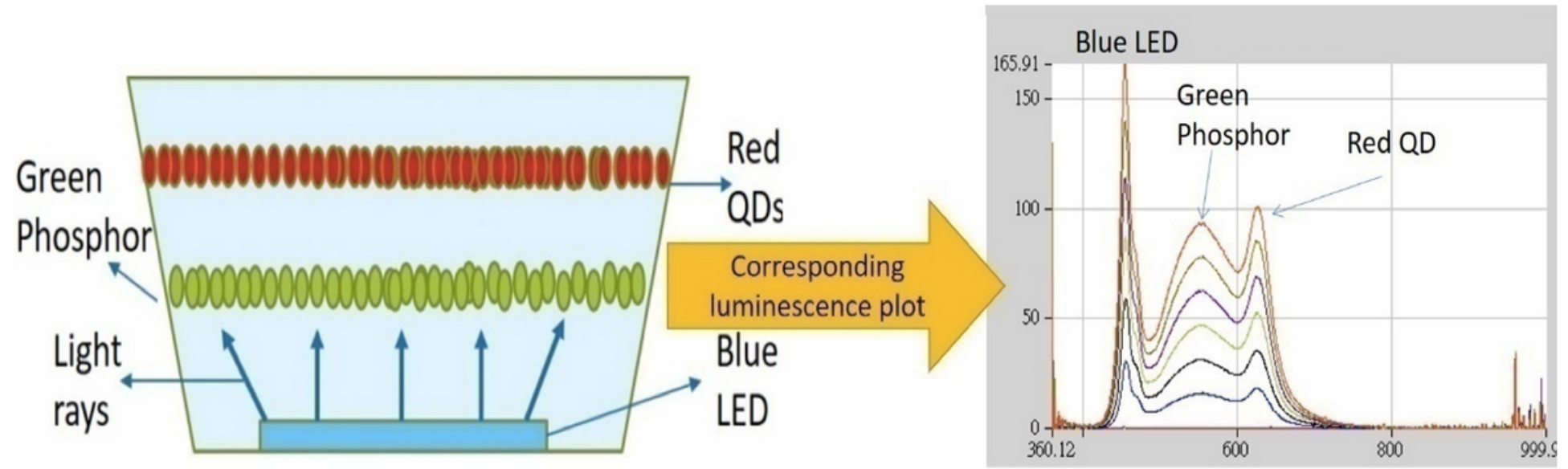

Figure 1

Schematic of LED structure and its corresponding luminescence plot. 


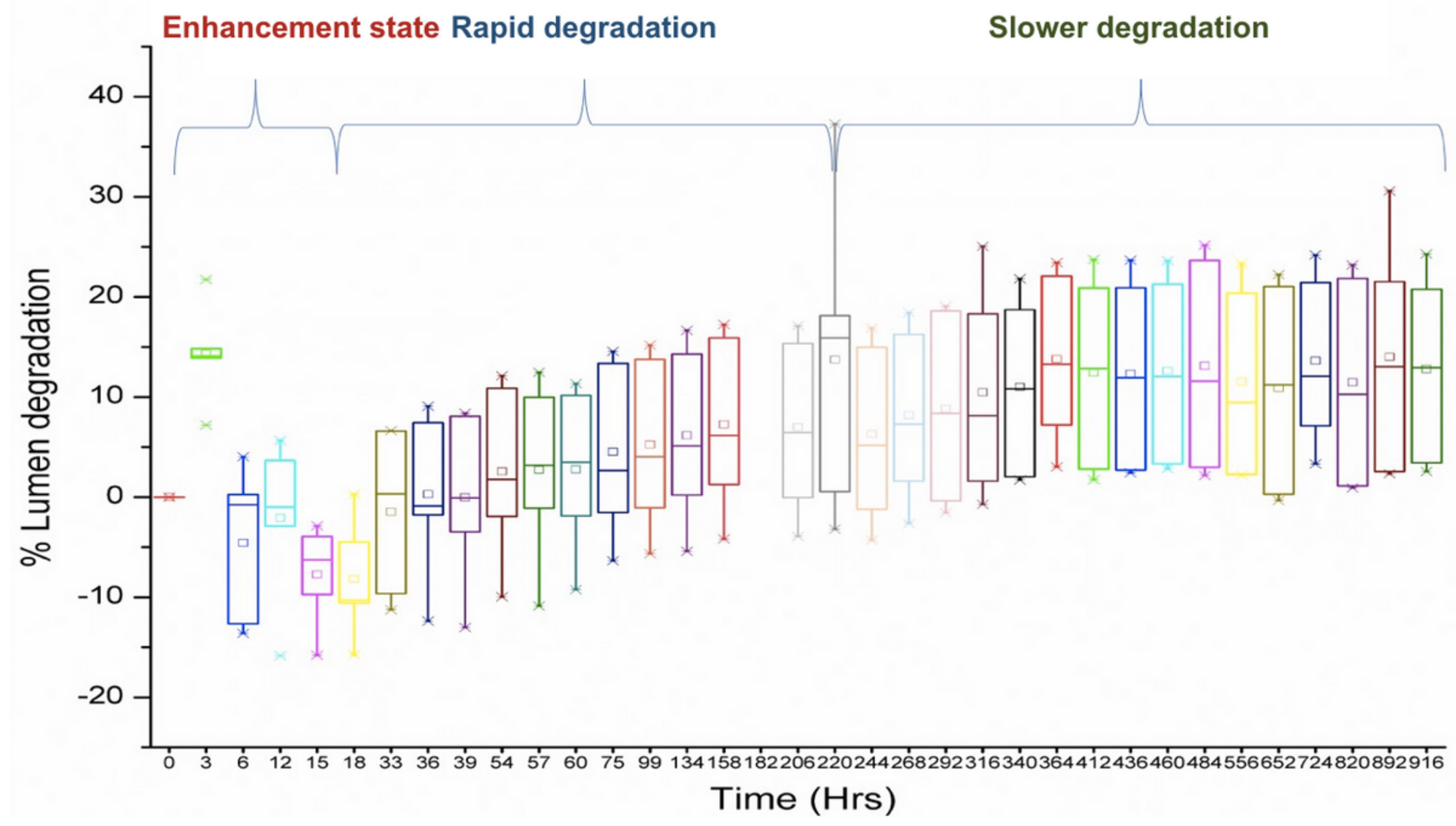

Figure 2

Overall LED luminescence variation with time.

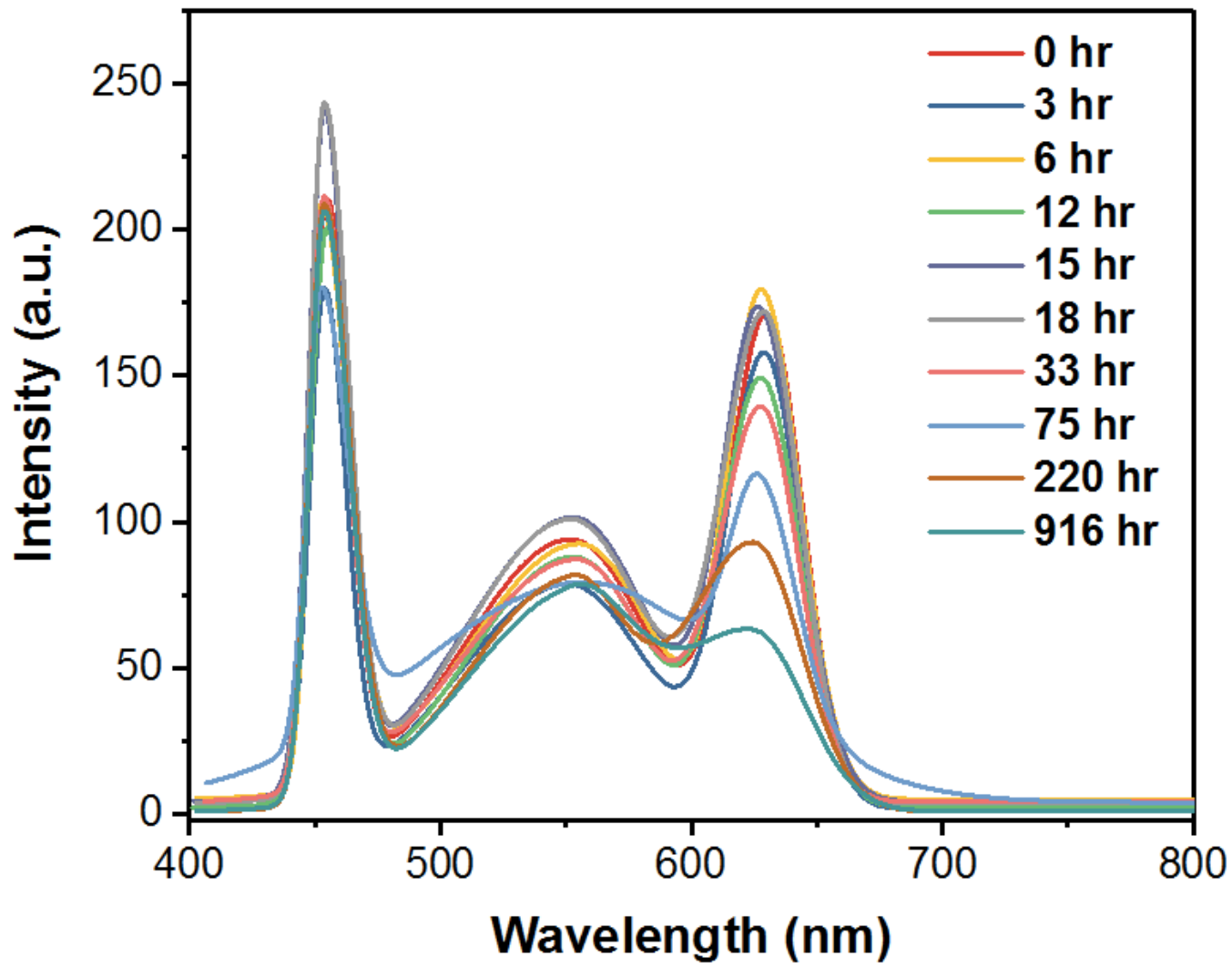

Figure 3

Overall packaged LED's luminescence data. 


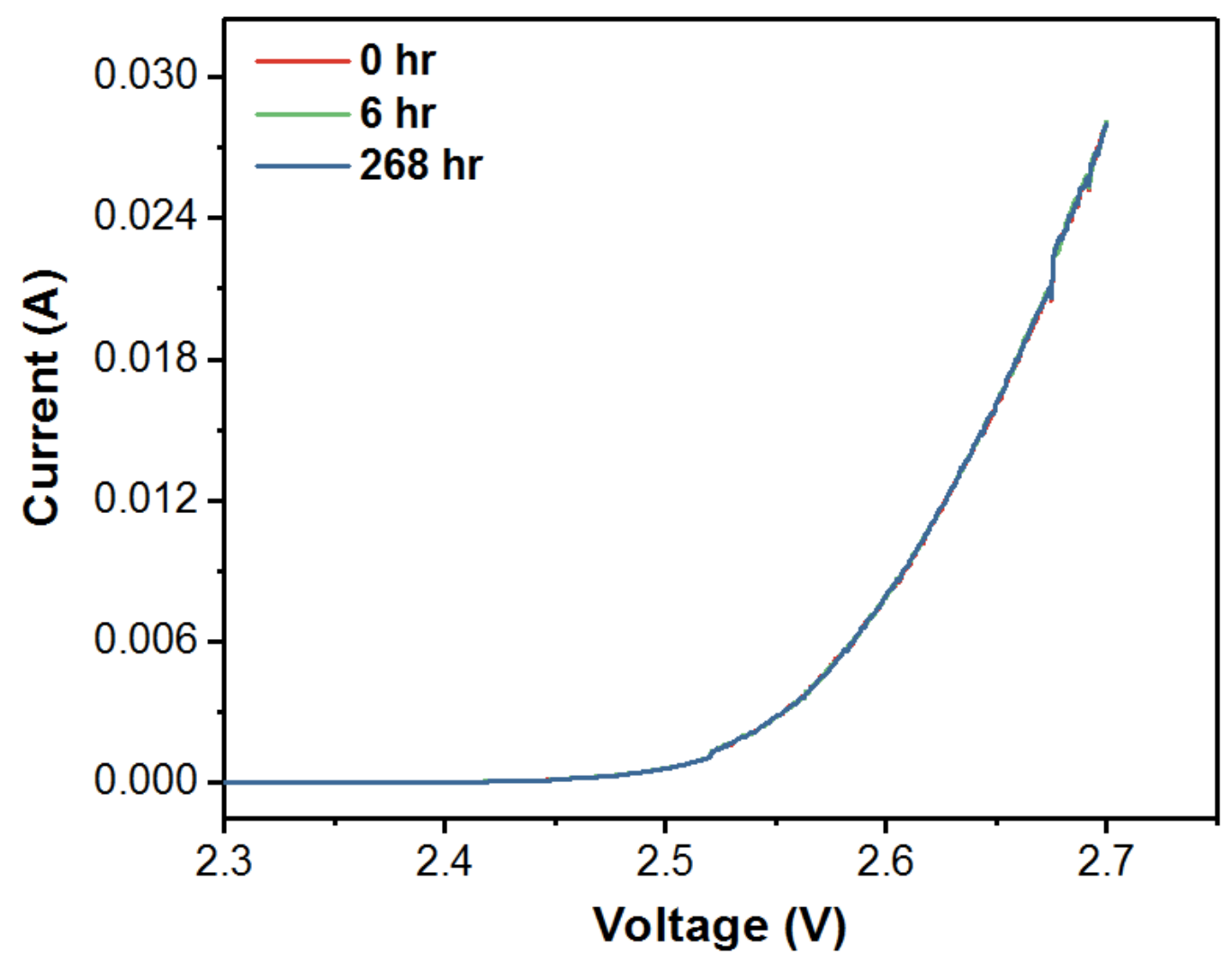

Figure 4

$\mathrm{I}-\mathrm{V}$ characteristics of LEDs tested under ambient conditions at different time intervals.

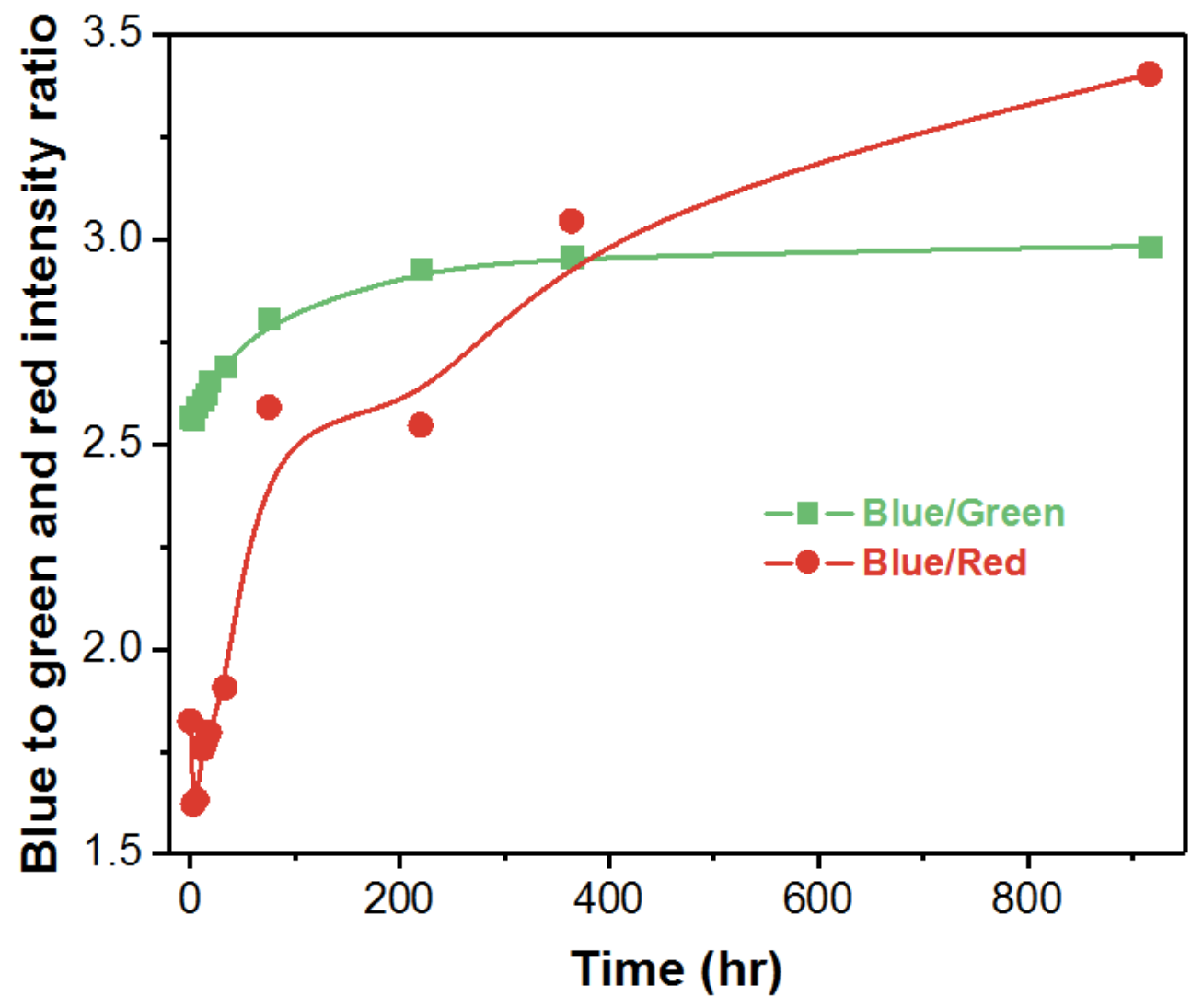

Figure 5

Comparison of light conversion efficiency for phosphor and QD at different time intervals in a packaged LED. 
(a)

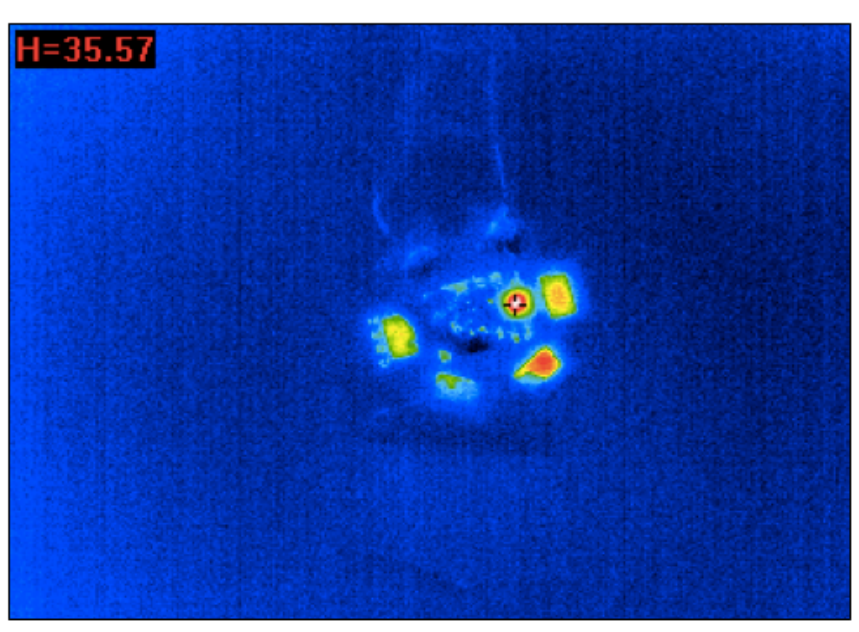

(b)

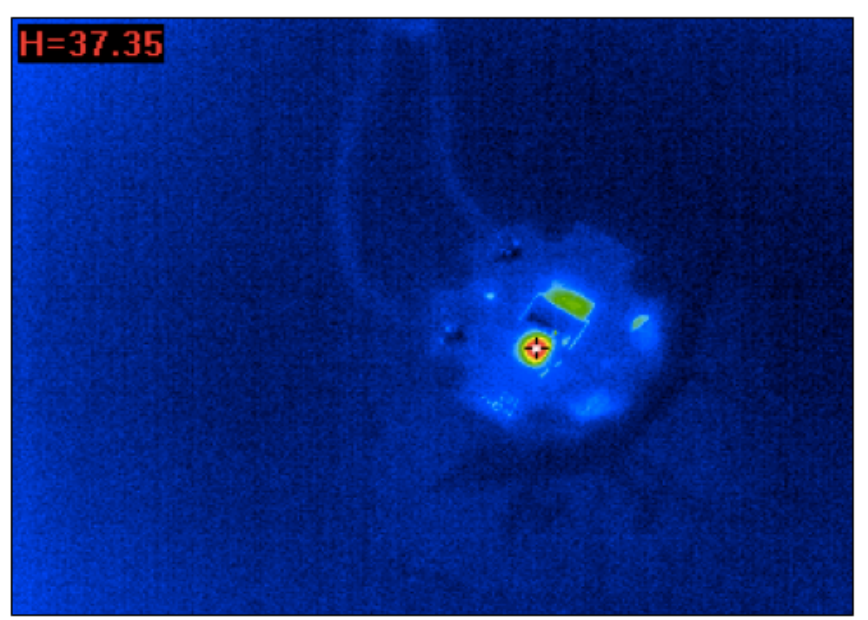

Figure 6

comparison between heat generation in LEDs (a) before and (b) after the test. Measurement is done using IR Camera (CHCT P384A-20 Thermal Scanning System)

Cd L $\alpha 1$

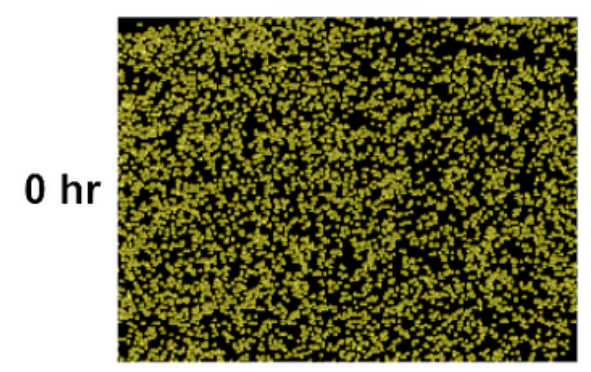

$18 \mathrm{hr}$
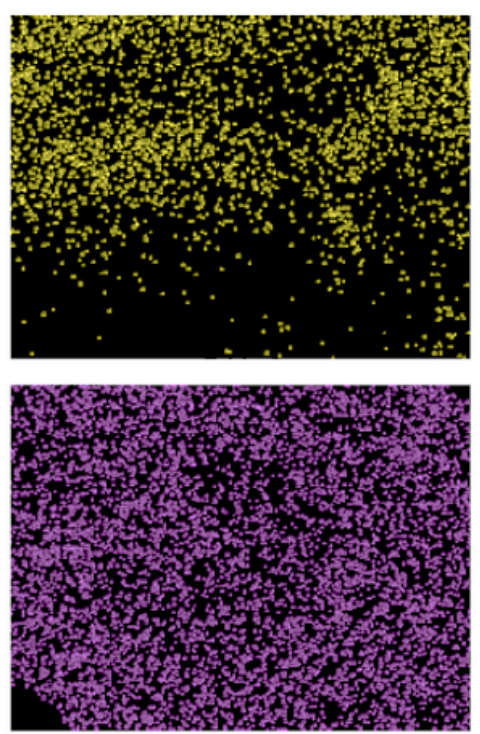

$\mathrm{S} K \alpha 1$
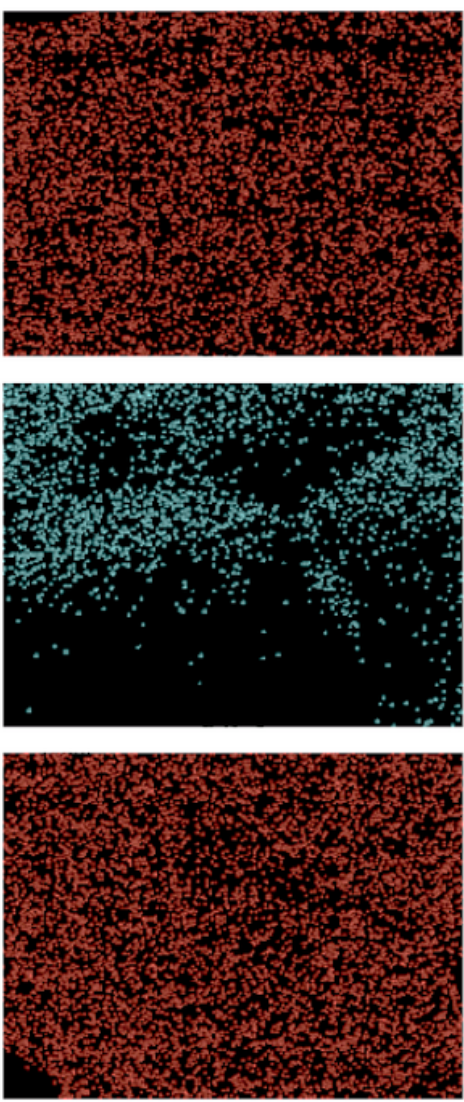

$\mathrm{Zn} \mathrm{K \alpha 1}$
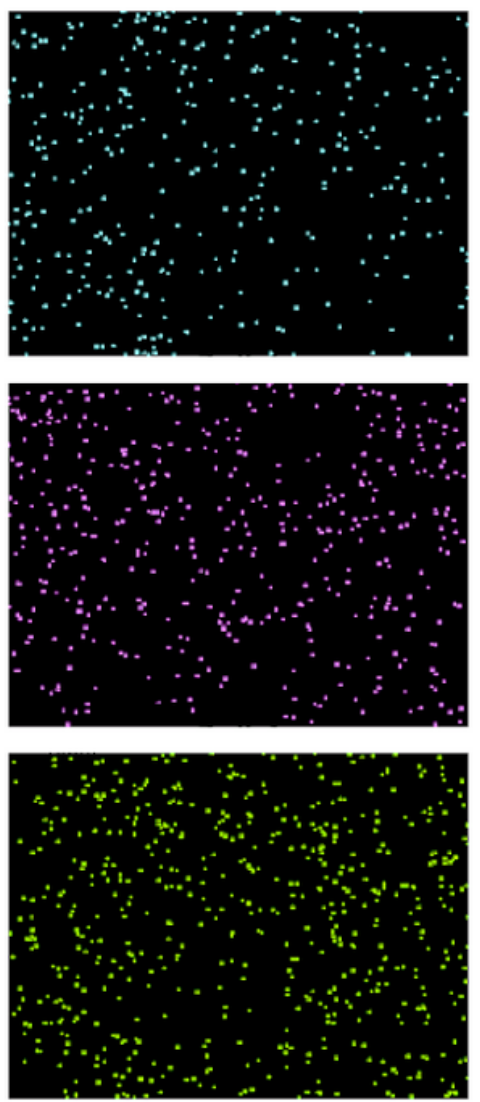

\begin{tabular}{|cc} 
& Weight (\%) \\
Cd & 52.2 \\
S & 22.6 \\
Zn & 5.2 \\
O & 20.0 \\
\hline
\end{tabular}

\begin{tabular}{cc} 
& Weight (\%) \\
Cd & 46.0 \\
S & 19.2 \\
Zn & 4.7 \\
O & 30.1 \\
\hline
\end{tabular}

\begin{tabular}{|cc|} 
& Weight (\%) \\
Cd & 42.5 \\
S & 19.0 \\
Zn & 4.3 \\
0 & 34.2 \\
\hline
\end{tabular}

Figure 7

The EDS mapping and element composition of QDs vs. test time tested under ambient condition. (Accelerating voltage $=10 \mathrm{kV}$, working distance $=5.7 \mathrm{~mm}$ ) 


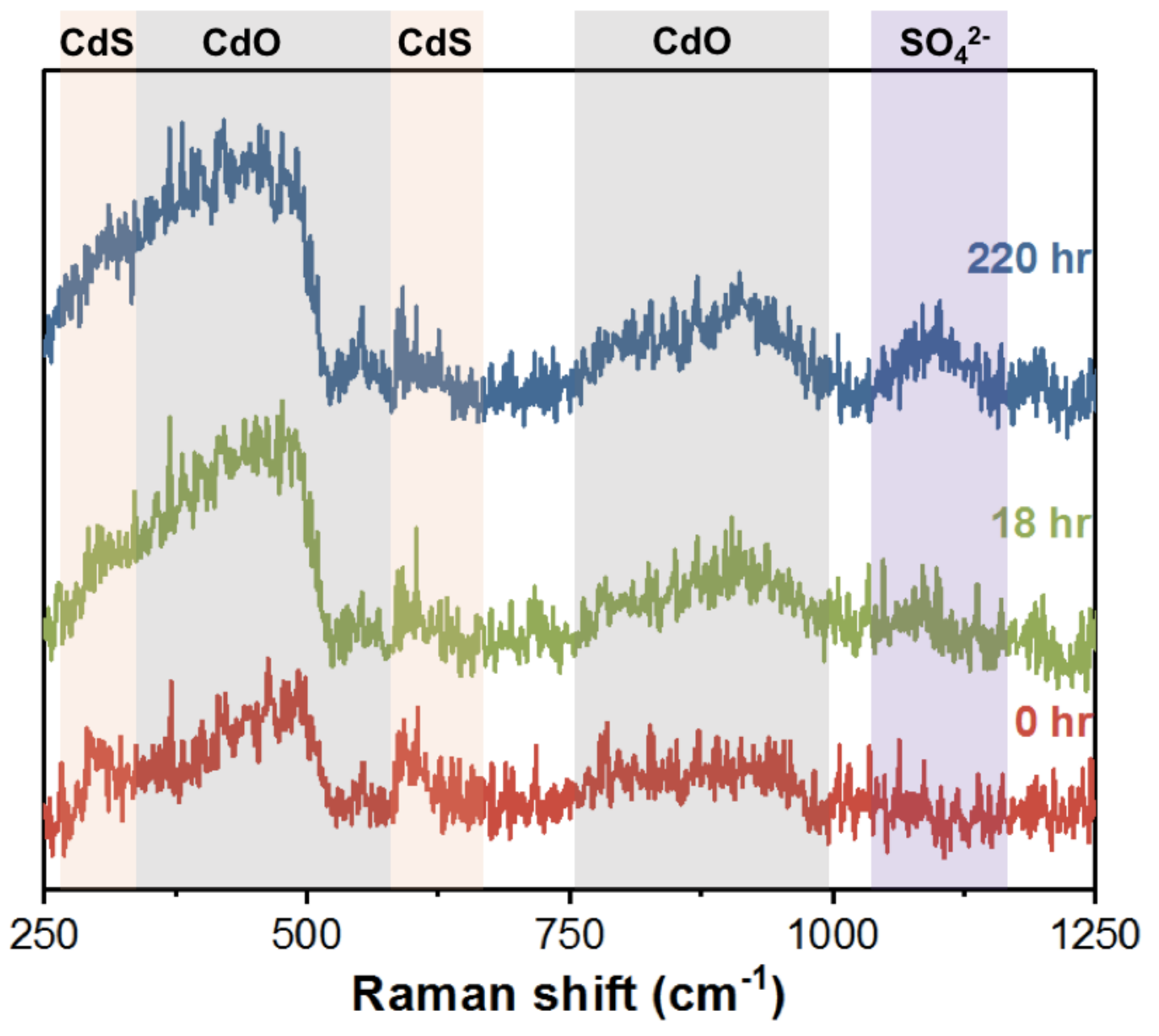

Figure 8

Raman spectra of QDs vs. test time tested under ambient condition. 


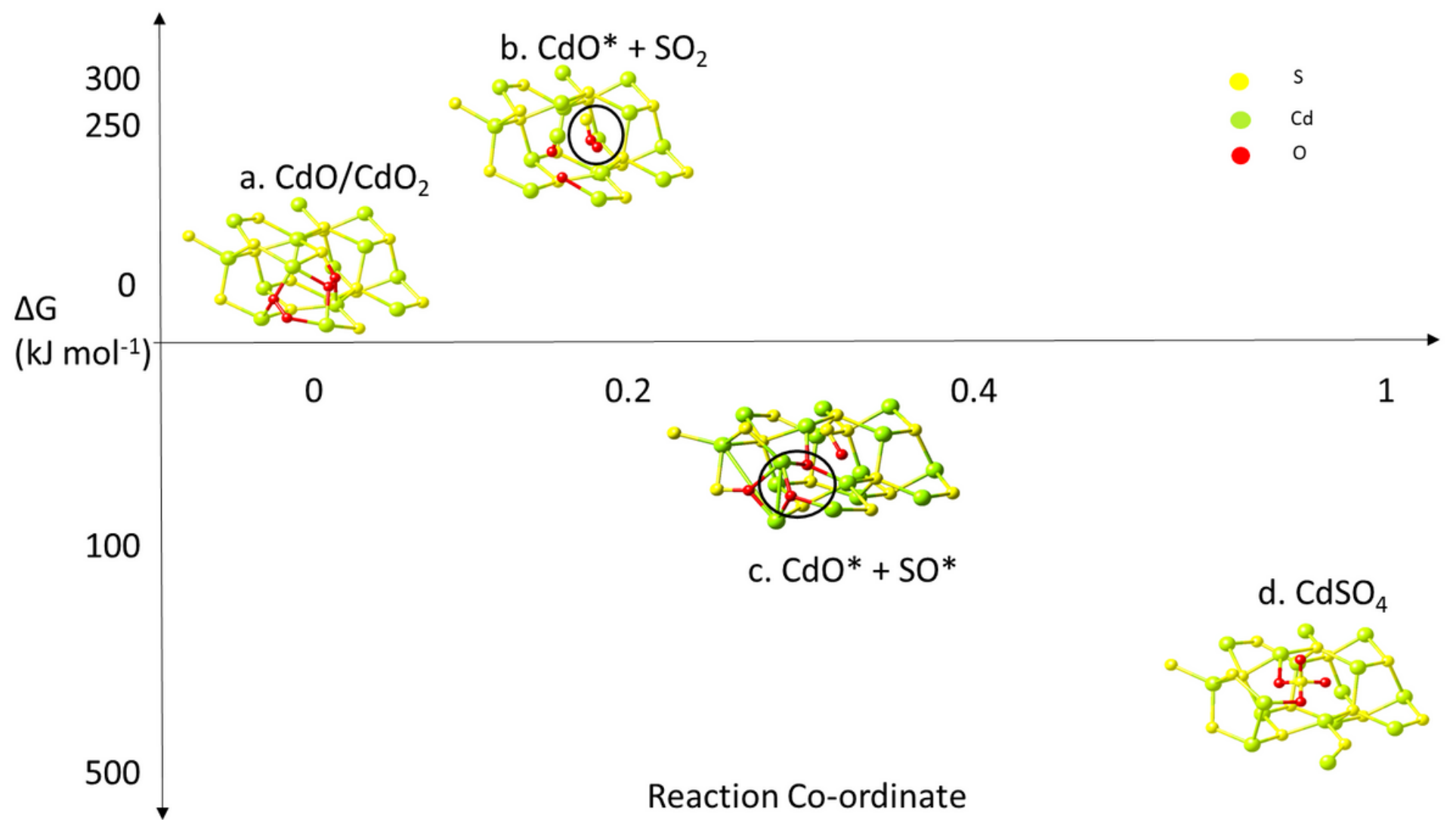

Figure 9

DFT Energy profile of $\mathrm{CdO}$ particles oxidizing into CdSO4. The black circles indicate the changes taking place in the reaction intermediates.

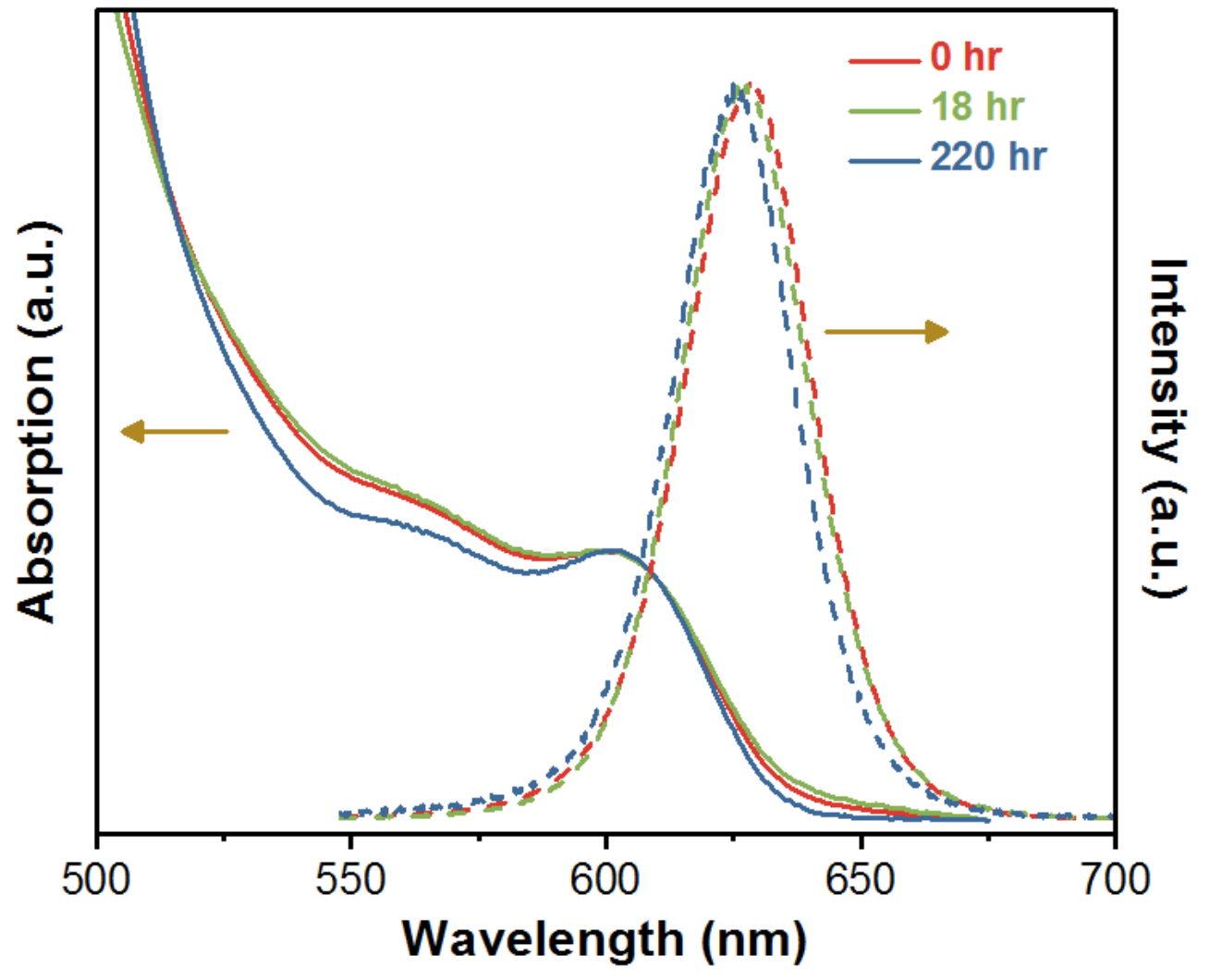

Figure 10

The absorption and PL spectra of QDs vs. test time tested under ambient condition. 


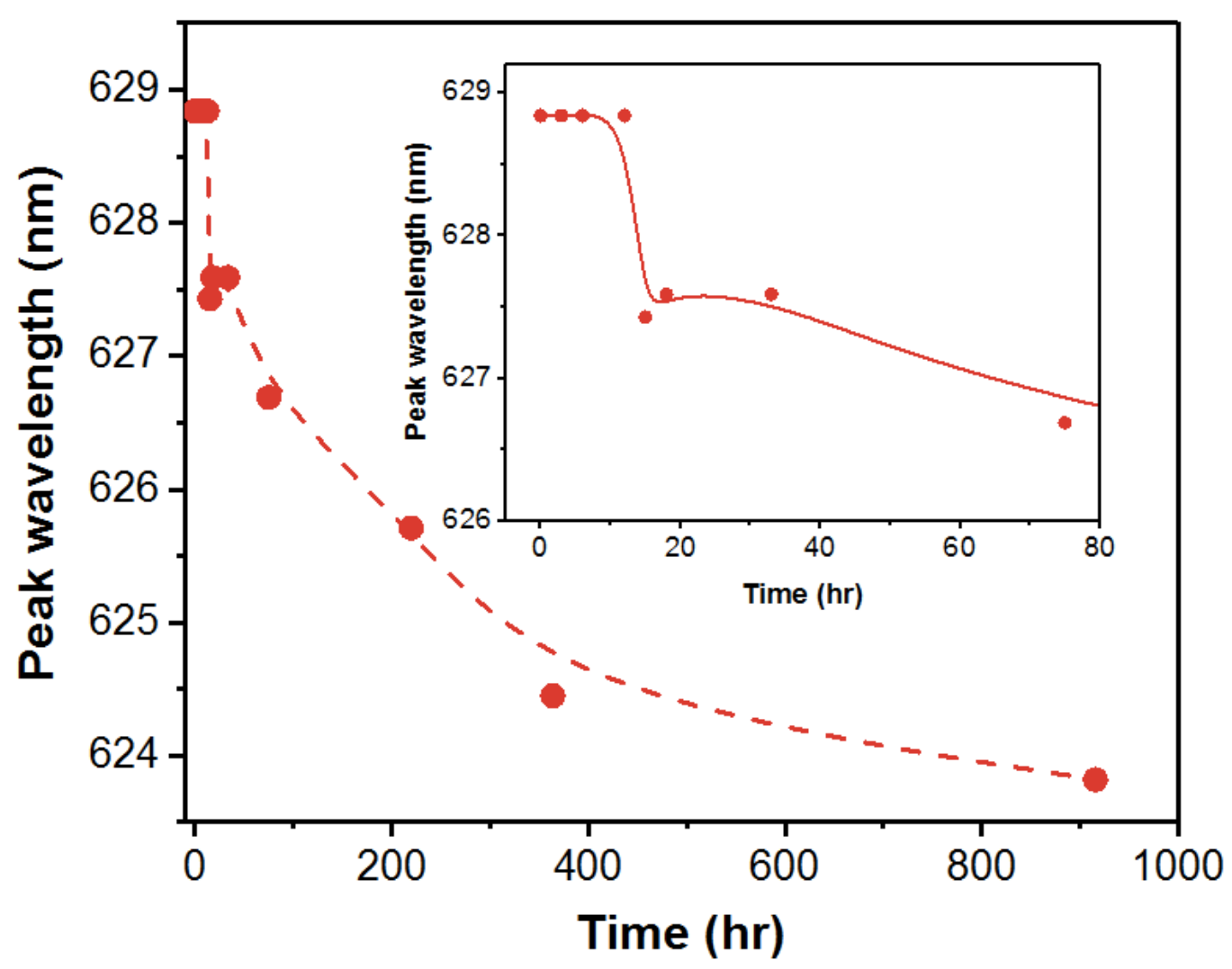

Figure 11

Average of variation of QDs peak wavelength vs. test time tested under ambient condition. 